\title{
A Design Methodology for Casting Ladle to Minimize Air Entrapment and Oxide Inclusion: An Application to Permanent Mold Casting Process
}

\author{
Jaehoon Choi ${ }^{1}$, Taekyung Kim ${ }^{1,2}$, Hoyoung Hwang ${ }^{3,4, *}$ and Shinill Kang ${ }^{1,2, *}$ \\ ${ }^{1}$ School of Mechanical Engineering, Yonsei University, 50 Yonsei-ro, Seodaemun-gu, Seoul 03722, Korea \\ ${ }^{2}$ National Center for Optically-assisted Mechanical Systems, Yonsei University, Seoul 03722, Korea \\ ${ }^{3}$ Digital Manufacturing Process Group, Korea Institute of Industrial Technology, \\ 113-58 Seohaean-ro, Siheung-si, Gyeonggi-do 15014, Korea \\ ${ }^{4}$ Industrial Materials and Smart Manufacturing Engineering, University of Science and Technology, \\ 217 Gajeong-ro, Yuseong-gu, Daejeon 34113, Korea
}

In this study, we proposed a design methodology for a ladle with flow characteristics that minimize air entrapment and oxidation during the pouring of molten aluminum in the casting process, and experimentally analyzed how filling behavior affects the porosity and mechanical properties of the cast product. To confirm the validity of the proposed ladle design, we performed a numerical analysis based on the solution algorithm for transient fluid flow to investigate mold filling behavior using the proposed ladle. We fabricated a ladle based on the design, and gravity cast the test specimens. Computed tomography was used to analyze the effects of the ladle's pouring characteristics on porosity. Tensile tests were performed to evaluate the mechanical properties of cast specimens. The designed ladle reduced casting porosity and improved the mechanical properties of the cast product. [doi:10.2320/matertrans.MT-M2019181]

(Received June 28, 2019; Accepted October 8, 2019; Published November 25, 2019)

Keywords: aluminum alloy, casting, mechanical properties, porosity, solution algorithm-volume of fluid

\section{Introduction}

Traditional aluminum alloys have excellent specific strength and are thus widely used lightweight materials in transport and machinery applications. Aluminum pieces are fabricated in permanent metal molds using various casting processes. $^{1-3)}$

In modern casting processes (e.g., die, evaporation, gravity casting and investment casting processes), such as that shown in Fig. 1(a) for die casting, molten metal oxidizes rapidly in air as it is poured into the cold chamber (Fig. 1(b)). Subsequently, the metal may be subjected to considerable splashing, which induces surface turbulence (Fig. 1(c)).

This turbulence introduces an entrained double oxide film or trail of buoyant bubbles in the metal and, as a result, casting defects (i.e., porosity) occur within the solidified metal. ${ }^{4-7)}$ Porosity defects can drastically degrade mechanical properties; pores also serve as paths for pressure leaks under pressurized conditions. Double oxide film defects form when the oxidized liquid metal surface is folded over onto itself and recombined with the bulk liquid. Thus, oxide inclusions are generated as a result of air entrainment. ${ }^{8-10)}$

Papworth et al. ${ }^{11)}$ examined that aluminum alloy castings are severely deteriorated by the aluminum oxide inclusions within the casting. ${ }^{12)}$ They also stated that oxide inclusions in the casting induce tertiary creep, pitting corrosion, stress, cracking. Although the effects of double oxide film can be reduced by expensive post-casting operations such as hot isostatic pressing, casting conditions leading to surface turbulence should be avoided if possible, as described by Nayhumwa et al. ${ }^{13)}$ Melendez et al. ${ }^{14)}$ also insisted that the removal of oxide inclusions from metal castings is required

*Corresponding authors, E-mail: snlkang@yonsei.ac.kr, hyhwang@ kitech.re.kr since the subsequent repair of those castings are expensive and time consuming.

Majidi et al. ${ }^{15)}$ insisted that air entrainment should be avoided, as it leads to oxidation inclusion in the final cast, which deteriorates the mechanical properties of the metal.

The effect of porosity defects on the mechanical properties of metal castings has been studied extensively. Caceres et al. ${ }^{16)}$ found out that the tensile properties of cast aluminum alloy are closely related to the area fraction of porosity and oxide films in the metal casting. Tensile strength deteriorates with an increase in the area fraction of defects, such as entrapped dross and oxide film defects, as well as with gas porosity in the fracture surface of samples. Dai et al. ${ }^{17)}$ investigated the effect of oxide film defects on the mechanical properties of aluminum-silicon-magnesium alloy castings; simulation and experimental results indicated fatigue crack generation in young oxide films, oxide films, and a combination of the two. Zeng et al. ${ }^{18)}$ found out that hydrogen in molten aluminum lead to pore formation in the castings. Aluminum oxide inclusion has a close correlation with the hydrogen content in aluminum melt.

Because the pouring conditions and gating system design have a major effect on air entrainment, and hence, oxide inclusion formation, there have been many studies on the effect of pouring conditions on air entrainment. The critical velocity of the melt front, which is about $0.5 \mathrm{~m} / \mathrm{s}$ for liquid aluminum, is a key factor in the suppression of surface turbulence in molten metals, as stated by Yang et al. ${ }^{19)}$

Several effective designs for gating systems have been proposed to control surface turbulence during pouring. Bozchaloei et al. $^{20)}$ investigated the effect of runner height on double oxide film formation during Al alloy casting; they confirmed that runner height plays a major role in controlling mechanical properties, such as ultimate tensile strength (UTS) and elongation. Additionally, their results indicated 
(a)

(b)

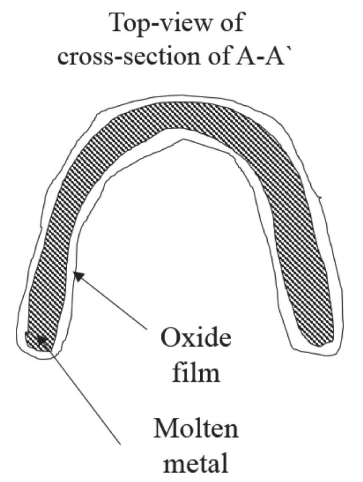

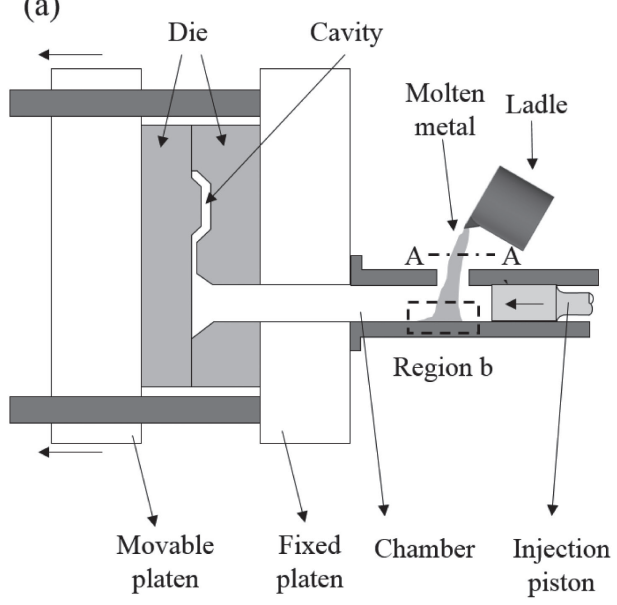

(c) Region b

Fig. 1 (a) Schematic diagram of die casting, (b) cross-section of the rapidly oxidized molten metal stream during pouring process by a ladle, and (c) entrained double oxide film and air bubbles produced by turbulent flow of molten metal. ${ }^{24)}$

that increasing the runner height from 12 to $24 \mathrm{~mm}$ decreased the UTS and elongation considerably.

The effect of the cross-sectional shape of runners on the mechanical strength of $\mathrm{Al}$ alloy has also been investigated in the study of Dai et $a l .{ }^{21)}$ using computational fluid dynamics modeling and experimental validation. Among three runner system designs (vortex-flow, rectangular, and triangular runner systems), the vortex-flow runner was most effective at minimizing the turbulent characteristics of molten metal flow in the runner by reducing the ingate velocity.

While the importance of gating systems in metal casting has been emphasized, the shape of the pouring lip of the ladle has received less attention. When metal is poured, it first travels across and through the ladle lip, before being transferred to the gating system and into the mold cavity. Less oxidation is always beneficial because the extent of the oxidation area contributes to oxide inclusion in the final product, which dramatically affects the mechanical properties. Thus, it is important to ensure that oxidation is not introduced in the first place.

In this paper, we introduce a ladle design with a pouring lip that can minimize air inclusion while pouring molten aluminum metal. The ladle lip was developed to minimize the surface area of the molten metal stream when falling from the lip. The proposed ladle design allows the cross-section of the molten metal stream to retain a small circular shape; thus, the air contact surface is relatively small compared with the cross-section of the molten metal stream poured by a conventional ladle. We conducted a numerical analysis based on a solution algorithm for transient fluid flow with multiple free boundaries, named SOLA-VOF developed by Nicholas et al., ${ }^{22)}$ to analyze air contact area with respect to the ladle design. We fabricated a ladle based on the proposed design and used it to fabricate test aluminum specimens via gravity mold casting. To confirm the validity of the proposed method, we analyzed the porosity fraction in the final cast using the computed tomography (CT), and subjected specimens to a tensile test. The proposed ladle design reduced the porosity of cast specimens and improved their mechanical properties.

\section{Experimental Methods}

\subsection{Numerical model of solution algorithm-VOF}

The continuity equation for modeling the flow of an incompressible fluid is as follows:

$$
\nabla \cdot V=0,
$$

where $V$ is the velocity vector. The volume of fluid (VOF) equation can be written as

$$
\frac{\partial F}{\partial t}+\nabla \cdot(V F)=0,
$$

where $t$ is time and $F$ is the fractional fluid volume. The Navier-Stokes equation is as follows:

$$
\frac{\partial V}{\partial t}+(V \cdot \nabla) V=G-\frac{1}{\rho} \cdot \operatorname{grad} P+\nu \nabla^{2} \cdot V,
$$


where $G, \rho, P$, and $v$ are the gravitational acceleration vector, density, pressure, and kinematic viscosity of the molten metal, respectively. The energy equation for an incompressible fluid is given by

$$
\rho c \frac{\partial T}{\partial t}-k \nabla^{2} T+\rho c V \cdot \operatorname{grad} \mathrm{T}+\rho L \frac{\partial f_{s}}{\partial t}=0,
$$

where $T, c$, and $L$ are the temperature, the specific heat of the fluid, and the latent heat, respectively, $f_{s}$ is the solid fraction, and $k$ is the thermal conductivity.

The VOF method uses the fractional volume function $F(x, y, z, t)$ to track free boundaries. When averaged over the cells of a computational mesh, the value of $F$ in a cell is equal to the fractional volume of the cell occupied by the fluid. $F=1$ corresponds to a cell full of fluid, whereas $F=0$ indicates that the cell contains no fluid. Cells with intermediate values contain a free surface.

$$
F=\frac{\text { Volume of fluid in a cell }}{\text { Volume of a cell }} .
$$

The VOF method considers the amount of $F$ to be fluxed through the face of a cell during the time-step duration, $\Delta \mathrm{t}$. The amount of $F$ in one time-step is $\delta F$ times the face crosssectional area, where

$$
\delta F=\operatorname{MIN}\left[F_{A D}\left|V_{x}\right|+C F, F_{D} \delta x_{D}\right],
$$

and

$$
C F=\operatorname{MAX}\left[\left(1.0-F_{A D}\right)\left|V_{x}\right|-\left(1.0-F_{D}\right) \delta x_{D}, 0.0\right] .
$$

The time increment necessary for stability is governed by several restrictions. The calculation converges when the following conditions are met:

$$
\Delta t<M I N\left\{\frac{\Delta x_{i}}{|u|}, \frac{\Delta y_{j}}{|v|}, \frac{\Delta z_{k}}{|w|}\right\}
$$

and

$$
\Delta t<\frac{3 \gamma}{4} \frac{\Delta x_{i}^{2} \cdot \Delta y_{j}^{2} \cdot \Delta z_{k}^{2}}{\Delta x_{i}^{2} \cdot \Delta y_{j}^{2}+\Delta y_{j}^{2} \cdot \Delta z_{k}^{2}+\Delta z_{k}^{2} \cdot \Delta x_{i}^{2}} .
$$

In the SOLA-VOF simulation, we compared the molten metal poured from a conventional ladle and the proposed ladle. The ladle used in the simulation was $\phi 84$ in diameter and $80 \mathrm{~mm}$ in height, with a wall thickness of $2 \mathrm{~mm}$; the fabricated ladles had the same dimensions. The ladles were filled $70 \%$ with molten aluminum AC3A alloy. The pouring temperature was $720^{\circ} \mathrm{C}$. The ladle was placed above the top of the mold and tilted for $3 \mathrm{~s}$ from $0^{\circ}$ to $100^{\circ}$ with respect to the mold's center. The ladles were tilted by $6 \mathrm{deg}$ every $0.2 \mathrm{sec}$ to have a constant angular velocity of $0.524 \mathrm{rad} / \mathrm{s}$.

\subsection{Fabrication of aluminum specimens for tensile test}

We fabricated ladles of two different shapes according to the conventional and proposed designs (diameter: $84 \mathrm{~mm}$; height: $80 \mathrm{~mm}$; wall thickness: $2 \mathrm{~mm}$ ). The two ladles were then used to make tensile test aluminum specimens via gravity mold casting under the same conditions as the simulation. We have fabricated ten test specimens, five of which were made of the proposed ladle, and the other five were made of the conventional ladle. Tensile test specimens made by casting were machined. In the tensile tests, an MTS
810 universal testing machine was used, and the tensile test conditions were set at $6 \mathrm{~mm} / \mathrm{min}$.

\subsection{Computed tomography analysis of cast specimens}

Industrial CT is a noninvasive computer imaging technique to analyze several cross-sectional X-rays in order to reveal minute details within a specimen as described in the study of Wits et $a .^{23)}$ A set of two-dimensional projection images is obtained during a full rotation of a specimen and subsequently three-dimensional images are reconstructed from two-dimensional serial computerized tomographic scan data. The reconstructed three-dimensional images of the specimen consist of volumetric pixels (voxels). In this study, the CT scans were performed with RayScan 250 (Rayscan, Germany). The voltage and current of X-ray sources were $180 \mathrm{kV}$ and $290 \mu \mathrm{A}$, respectively. The voxel sizes were $276.41 \mu \mathrm{m}$.

\section{Results and Discussion}

\subsection{Mold filling behaviors with the proposed ladle}

Figure 2 shows a comparison of simulated mold filling behaviors when using the conventional and proposed ladle. The conventional ladle typically has a sharp tip, as shown in Fig. 2(a). Figure 2(b) shows the mold filling simulation result using the conventional ladle. In this case, the molten metal splashes due to surface turbulence upon reaching the mold (or shot sleeve of cold chamber high pressure die casting).

Subsequently, the surface oxide film often breaks up before entering the molten metal, as shown in Fig. 2(c). Figure 2(d) shows the cross-section of the A-A' plane. In contrast to the conventional ladle, the proposed ladle has a rounded tip, designed to provide gentle outflow characteristics, as shown in Fig. 2(e). The dimensions of the proposed ladle were chosen according to design of experiments to minimize surface turbulence. The rounded configuration also guarantees a low free-fall height, thus avoiding surface turbulence as the molten metal is being poured (Fig. 2(f)). The proposed ladle provides gentle outflow characteristics in contrast to the conventional ladle, minimizing surface turbulence and air entrapment as shown in Fig. 2(g).

\subsection{Comparison of air contact area}

As a result, the molten metal is introduced to the mold such that the oxide film only forms at the end of the metal flow. Figure 2(h) shows the cross-section of the B-B' plane. Figure 3(a) and 3(b) compare the mold filling behavior of molten aluminum when pouring using the conventional and proposed ladle at a tilting time of $2.0 \mathrm{~s}$. Figure 3(c) shows the $\mathrm{V}$-shaped cross-section of the molten metal being poured, as well as the separation of a portion of the molten metal from the main stream. In free surface flows, the air is easily entrained with this type of surface discontinuity.

Using the proposed configuration of the ladle, the molten metal stream retained a circular-shaped cross-section, without any stream separation (Fig. 3(d)). Given that the oxidation induces oxide inclusion defects in the final casting, minimization of the air contact area is important. The air contact area is defined by the number of cells exposed to air in the cross-section of the simulated molten metal stream, as shown in Fig. 4(a). From the comparison, it can be seen that 
(a)

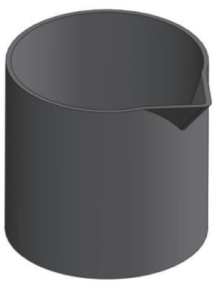

(e)

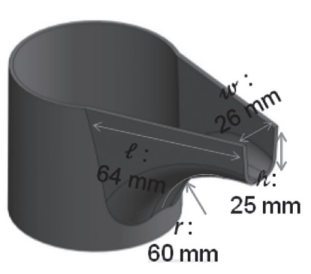

(f) (b)
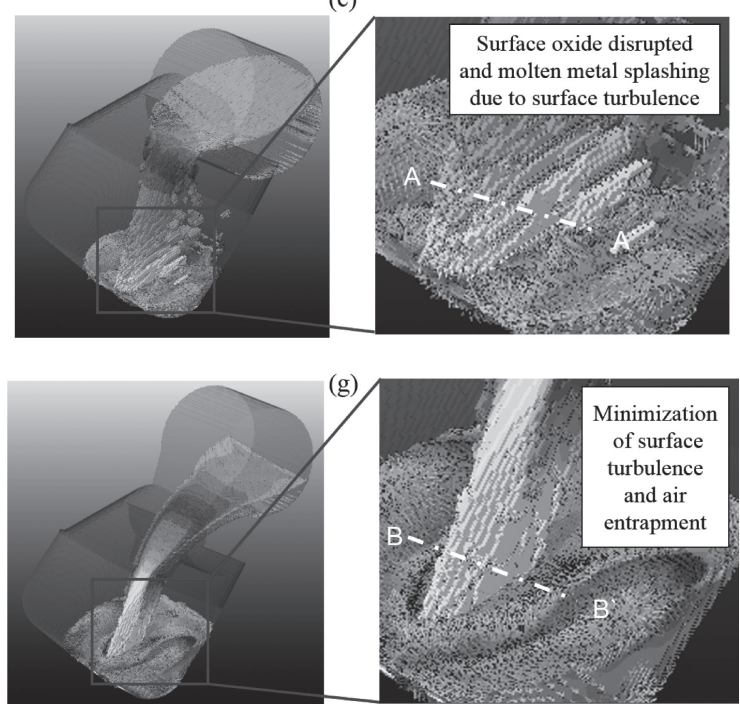

(d)

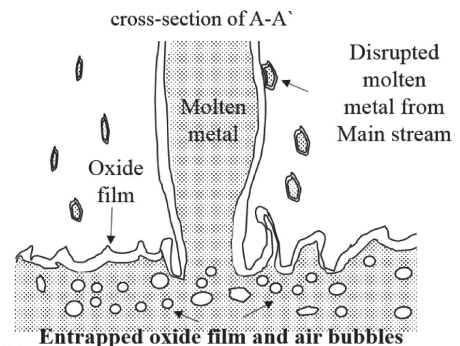

(h)

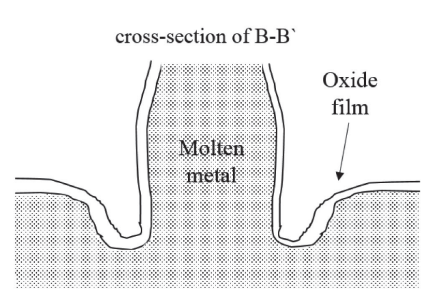

Fig. 2 (a) A conventional ladle, (b) mold filling simulation by the conventional ladle, (c) surface oxide disruption and molten metal splashing due to surface turbulence when mold filling using the conventional ladle, (d) cross-section of A-A', (e) the proposed ladle with the designed dimensions (f) mold filling simulation using the proposed ladle, (g) minimization of surface turbulence and air aspiration when mold filling using the proposed ladle, and (h) cross-section of B-B'.

(a)

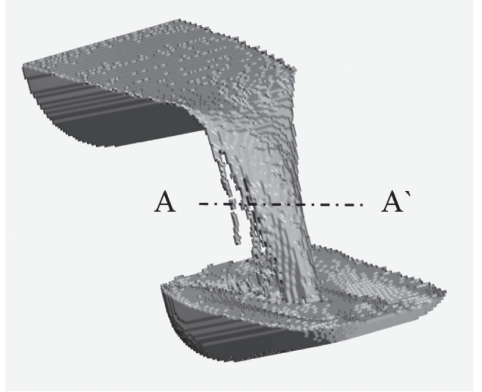

(c)

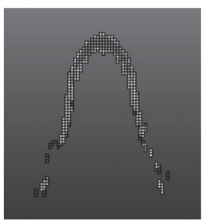

$1.0 \mathrm{~s}$

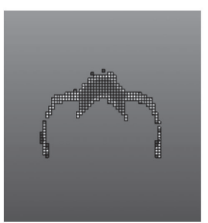

$2.0 \mathrm{~s}$

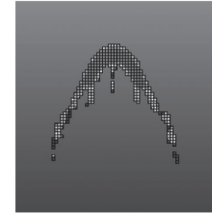

$1.5 \mathrm{~s}$

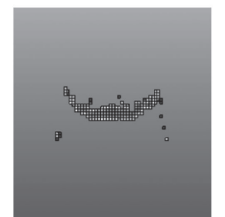

$2.5 \mathrm{~s}$

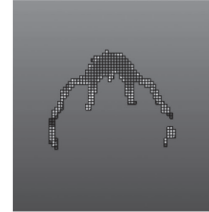

$2.0 \mathrm{~s}$

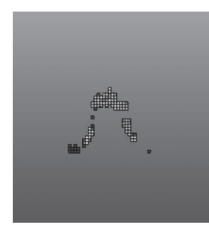

$3.4 \mathrm{~s}$

(b)

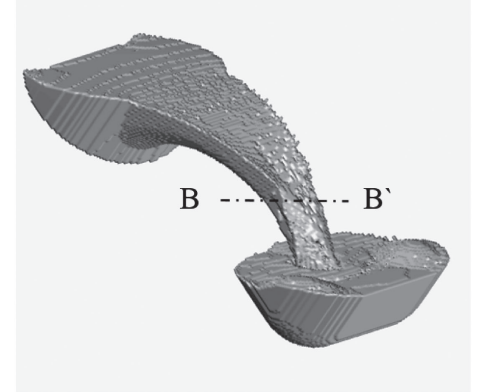

(d)

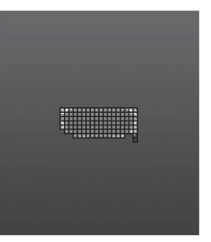

$0.5 \mathrm{~s}$

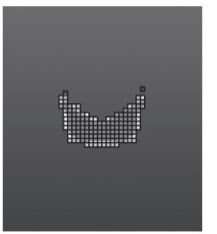

$2.0 \mathrm{~s}$

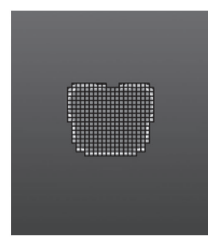

$1.0 \mathrm{~s}$

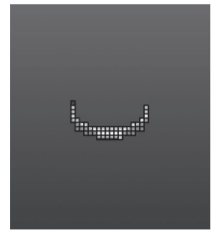

$2.5 \mathrm{~s}$

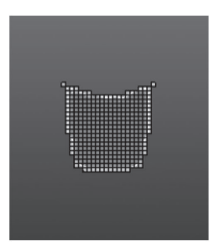

$1.5 \mathrm{~s}$

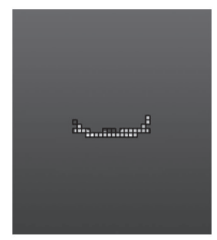

$3.0 \mathrm{~s}$

Fig. 3 Simulated mold filling behavior of molten aluminum when pouring using (a) a conventional ladle and (b) the proposed ladle for a tilting time of $2 \mathrm{~s}$. The cross-section of the molten metal stream according to tilting time using (c) a conventional ladle and (d) the proposed ladle.

the conventional ladle had a greater air contact area than that of the proposed ladle (Fig. 4(b)).

\subsection{Internal porosity of casted specimen}

Figure 5 shows industrial CT scans of tensile test aluminum specimens cast by the conventional and proposed ladles. The internal porosity was quantified by the volume of each pore. Once the volume of each pore was identified, the results were assigned a color according to the volume. Based on reconstructed three-dimensional CT images and two-dimensional (2D) slice images (Figs. 5(a) and 5(b), respectively), we confirmed that the cast specimen by the 
(a)

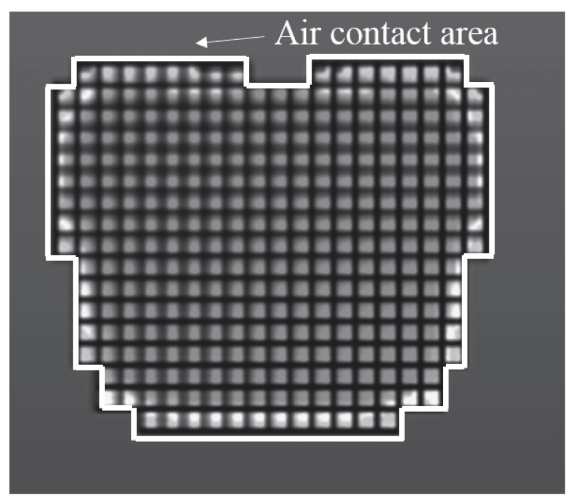

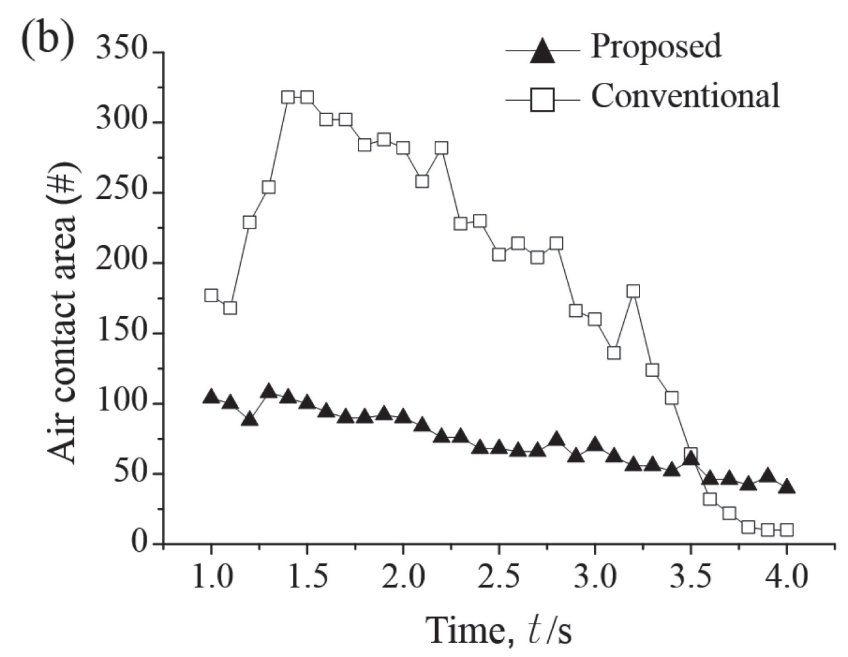

Fig. 4 Air contact area defined by the number of cells exposed to air in (a) the cross-section of the simulated molten metal stream and (b) comparison of the air contact area using the proposed and conventional ladle.

(a)

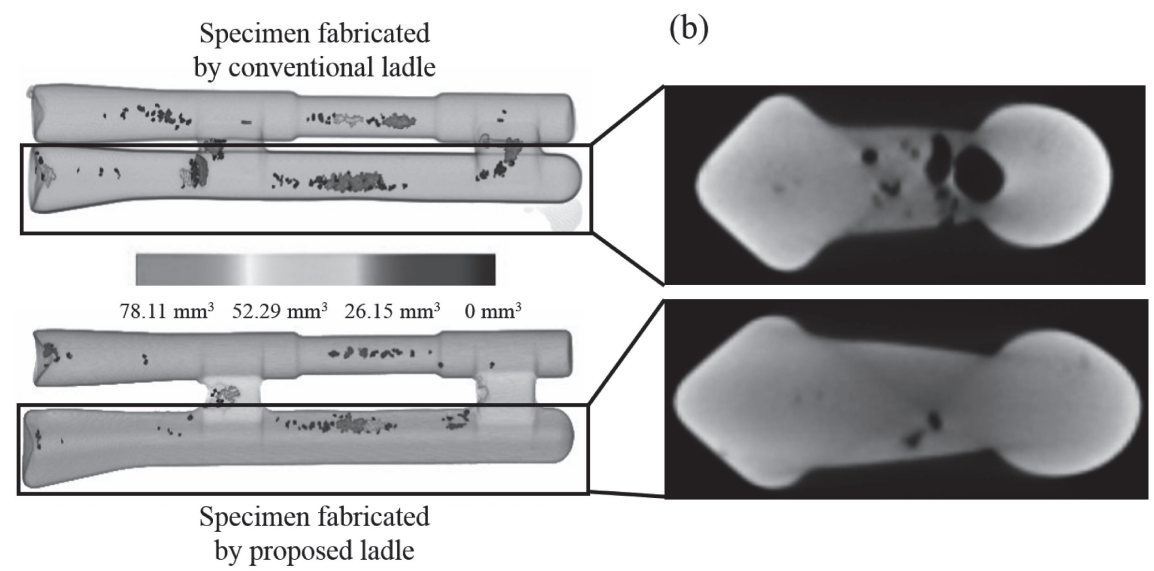

(c)

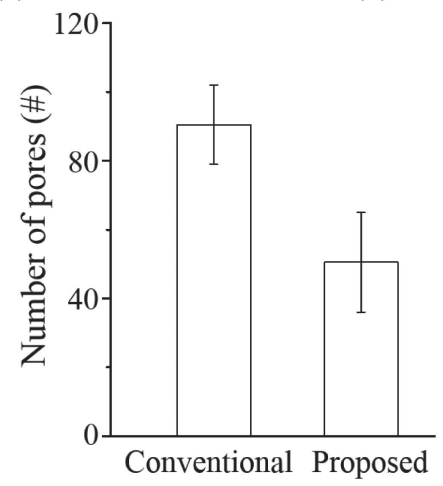

(d)

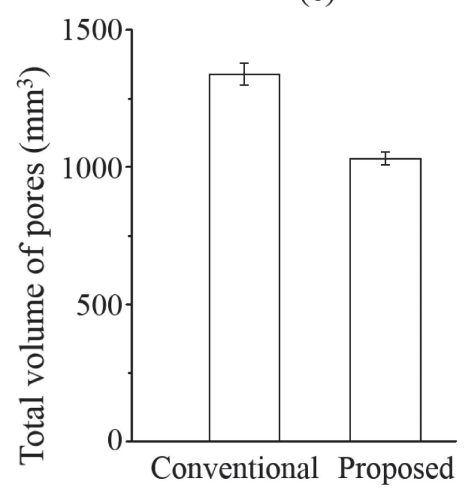

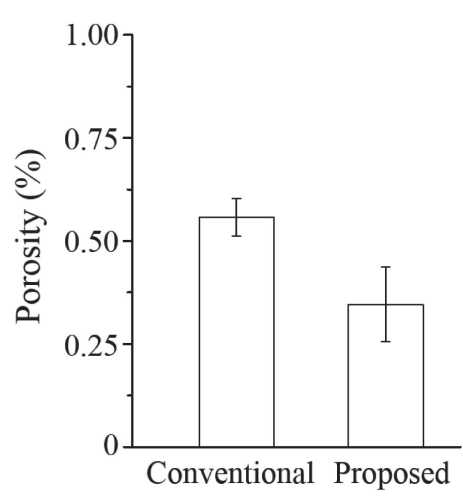

Fig. 5 Industrial CT scanning images and analysis of internal porosity. (a) Reconstructed three-dimensional CT images of cast specimens by the conventional and proposed ladles, and (b) two-dimensional (2D) CT slice images. The color bar indicates the volume of each pore in cubic micrometers. The black portion in the 2D images indicates the internal pores inside the specimens. Analysis of (c) the number of internal pores, (d) the total volume of the internal pores, and (e) the internal porosity, based on CT scanning images.

proposed ladle possessed fewer internal pores. Figure 5(a) shows the pore volume in cubic micrometers, using a color bar representation. In 2D slice images, the black portion indicates the internal pores inside the specimens. Figure 5(c)-5(e) shows the number of internal pores, the volume of the internal pores, and the internal porosity of cast specimens, respectively. The specimen cast with the conventional ladle had an average of 90.5 pores, compared with an average of 50.5 pores using the proposed ladle design.
Use of the conventional ladle resulted in a total internal pore volume of $1,338.8 \mathrm{~mm}^{3}$, versus $792.1 \mathrm{~mm}^{3}$ for the proposed ladle. The average internal porosity using the conventional ladle was $0.557 \%$, versus $0.3455 \%$ with the proposed ladle. The error bars in the graphs represent the standard deviation of the experiments. Thus, specimens cast with the proposed ladle were expected to demonstrate better performance in tensile tests. 
(a)

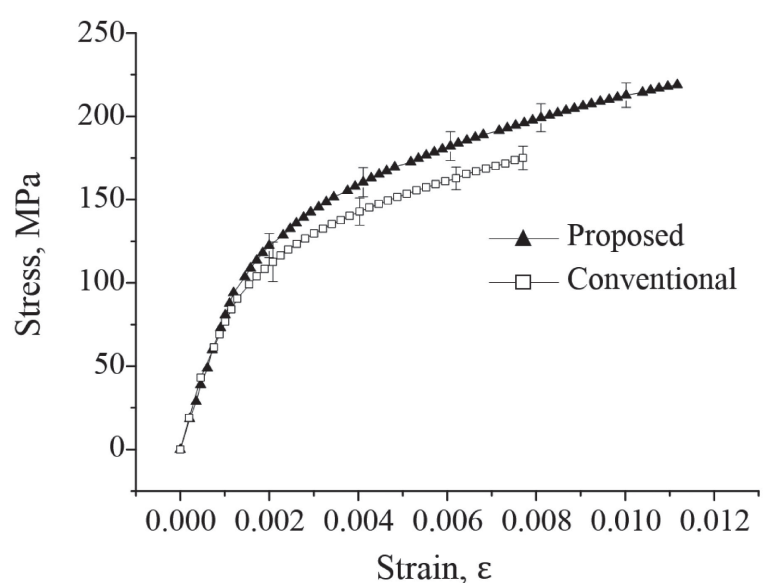

(b)

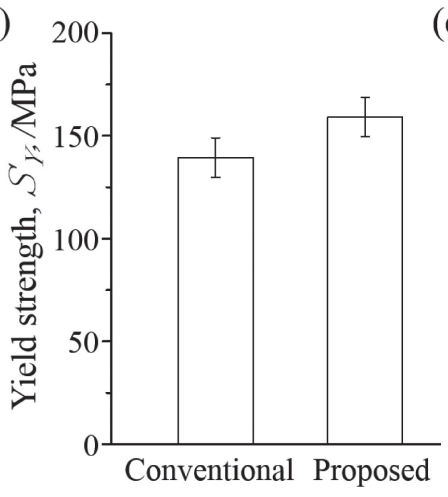

(c)

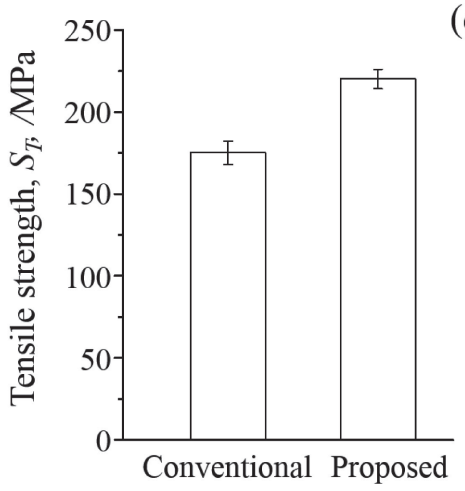

(d)

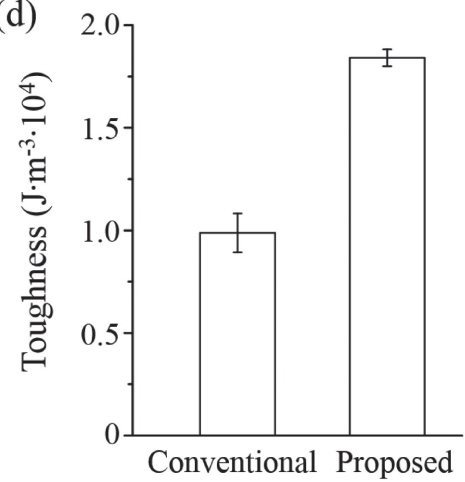

Fig. 6 Tensile test results using an MTS 810 universal testing system. Comparison of (a) stress-strain curves, (b) yield strength, (c) tensile strength, and (d) toughness of cast specimens using the proposed versus conventional ladle configurations.

\subsection{Mechanical properties of cast specimens based on analysis of tensile test results}

Figure 6 compares the tensile test results for specimens cast using the conventional and proposed ladle. Figure 6(a) shows the stress-strain curves of the cast specimens. The specimens cast by the proposed ladle showed higher yield strength, tensile strength, and toughness.

Specifically, the specimens made using the conventional ladle had a yield strength and average tensile strength of 139.467 and $175.039 \mathrm{MPa}$, respectively; in contrast, the specimens produced with the proposed ladle demonstrated a yield strength and average tensile strength of 159.262 and 220.157 MPa, respectively (Figs. 6(b) and 6(c)). The specimens made using the conventional ladle had an average toughness of $0.987 \times 10^{4} \mathrm{~J} \cdot \mathrm{m}^{-3}$, versus $1.840 \times 10^{4} \mathrm{~J} \cdot \mathrm{m}^{-3}$ using the proposed ladle, as shown in Fig. 6(d). The error bars in the graphs represent the standard deviation of the experiments.

\section{Conclusion}

We proposed a design methodology for a ladle with flow characteristics that minimize air entrapment and oxidation inclusions. The numerical analysis based on SOLA-VOF revealed that the surface turbulent transfer of molten metal was minimized, and air contact area was also reduced when using the proposed ladle. To validate the design methodology, internal porosity and mechanical properties of casted specimens were analyzed. Compared with the conventional ladle, the proposed ladle resulted in an average of 40 fewer pores, an average total pore volume that was $307.1 \mathrm{~mm}^{3}$ smaller, and an average difference in porosity of $0.21 \%$. A comparison of the results of tensile tests revealed that specimens made using the proposed ladle had average increases in yield strength, tensile strength, and toughness of $19.795 \mathrm{MPa}, 45.118 \mathrm{MPa}$, and $0.853 \mathrm{~J} \cdot \mathrm{m}^{-3} \cdot 10^{4}$, respectively.

\section{Acknowledgements}

This work was supported by the National Research Foundation of Korea (NRF) (2015R1A5A1037668) and the Ministry of Trade, Industry and Energy (Grant N0002310).

\section{REFERENCES}

1) Y. Iwata, D. Shuxin, Y. Sugiyama and H. Iwahori: Mater. Trans. 55 (2014) 311-317.

2) K.L. Fan, X.S. Liu, G.Q. He and H. Chen: Mater. Sci. Eng. A 632 (2015) 127-136.

3) M. Hino, K. Murakami, N. Nagata, C. Ibata, H. Kanetsuki and S. Kawai: Mater. Trans. 54 (2013) 2238-2244.

4) Y. Hangai, S. Kitahara and S. Amada: Mater. Trans. 47 (2006) 23632367.

5) X.G. Hu, Q. Zhu, S.P. Midson, H.V. Atkinson, H.B. Dong, F. Zhang and Y.L. Kang: Acta Mater. 124 (2017) 446-455.

6) M. Tiryakioğlu: Mater. Sci. Eng. A 465 (2007) 287-289.

7) S.H. Mousavi Anijdan and M. Sabzi: Mater. Sci. Eng. A 737 (2018) 230-235.

8) N. Omura, Y. Murakami, M. Li, T. Tamura, K. Miwa, H. Furukawa, M. Harada and M. Yokoi: Mater. Trans. 50 (2009) 2578-2583.

9) T.R. Vijayaram, S. Sulaiman, A.M.S. Hamouda and M.H.M. Ahmad: J. Mater. Process. Technol. 178 (2006) 29-33. 
10) S. Dong, Y. Iwata, Y. Sugiyama and H. Iwahori: Mater. Trans. 51 (2010) 371-376.

11) A. Papworth and P. Fox: Mater. Lett. 35 (1998) 202

12) J. Campbell: Mater. Sci. Technol. 22 (2006) 127.

13) C. Nayhumwa, N.R. Green and J. Campbell: Metall. Mater. Trans. A 32 (2001) 349-358.

14) A.J. Melendez, K.D. Carlson and C. Beckermann: Int. J. Cast. Met. Res. 23 (2010) 278-288.

15) S.H. Majidi and C. Beckermann: Int. J. Cast. Met. Res. 30 (2017) $301-$ 315.

16) C.H. Cáceres and B.I. Selling: Mater. Sci. Eng. A 220 (1996) 109-116.

17) X. Dai, X. Yang, J. Campbell and J. Wood: Mater. Sci. Technol. 20 (2004) 505-513.
18) J. Zeng, D. Li, M. Kang, H. He and Z. Hu: J. Nanosci. Nanotechnol. 13 (2013) 6948-6952.

19) X. Yang, X. Huang, X. Dai, J. Campbell and J. Tatler: Int. J. Cast. Met. Res. 17 (2004) 321-331.

20) G.E. Bozchaloei, N. Varahram, P. Davami and S.K. Kim: Mater. Sci. Eng. A 548 (2012) 99-105.

21) X. Dai, X. Yang, J. Campbell and J. Wood: Mater. Sci. Eng. A 354 (2003) 315-325.

22) B.D. Nichols, C.W. Hirt and R.S. Hotchkiss: Los Alamos Scientific Lab. No. LA (1980) 8355.

23) W.W. Wits, S. Carmignato, F. Zanini and T.H. Vaneker: CIRP Ann. 65 (2016) 201-204.

24) J. Campbell: Mater. Sci. Technol. 22 (2006) 127-145. 